\title{
The Possibility of Developing Geographic and Cartographic Education using Minecraft
}

\author{
Carla Cristina Reinaldo Gimenes de Sena ${ }^{\mathrm{a}, *}$, Barbara Gomes Flaire Jordão ${ }^{\mathrm{b}}$ \\ ${ }^{a}$ UNESP, Carla Cristina Reinaldo Gimenes de Sena - carla.sena@unesp.br \\ ${ }^{b}$ USP, Barbara Gomes Flaire Jordão - barbaraflaire@ hotmail.com \\ * Corresponding author
}

Keywords: Cartographic Education, Games, Minecraft

\begin{abstract}
:
The use of technology is closer and closer to the school, providing new perspectives to the geographic education, which includes the learning of cartography and learning through cartography as being important resources for the geographic space' analysis. As an example of digital technologies application, this work presents a reflection on the use of virtual environments for the learning diagnosis of geographic and cartographic concepts. Digital games based on construction draw children's and young people's attention, and among them, Minecraft is one of the most popular. This is a game that allows its user to explore a virtual world made of cubic blocks, which imitate vegetation, rock formations, water, among other elements, in different game modes. In creative mode, it is possible to create a personal world without barriers or challenges. On its turn, in survival mode, the player is inserted in a pre-created world, and to advance and create new environments it is necessary to overcome challenges. In the years of 2017, 2018 and 2019, about 500 students between 10 and 13 years old were invited to build, in creative mode, watersheds presented with different characteristics, in order to figure out their main elements and their relation to human development. During the sequence of activities, students were divided into groups up to five and they had to build the watershed randomly designated to them by lot, including the specific characteristics requested by the teacher. After the construction, elements such as point of view, proportion, and coordinates were used to present the final projects. In addition to the great participation in the activity, the work inside the game provided the application of fundamental concepts for the reading and interpretation of maps, besides those related to water resources.
\end{abstract}

From that experience, the research unfolded in proposing the same activity to a group of senior high school students. Four students between 16 and 18 years old were challenged to build, also in creative mode, an environment that reproduced a watershed. This experience used the Minecraft Windows 10 (Beta - 1.16.0.57) version, and in about four hours the environment with a watershed was developed. Due to the age range and game experience, it was remarkable the students' concern with the project's details, especially with the relation of proportion and seeking solutions for the stages of construction. In this way, it was possible to assess that the participants used their knowledge of scale, orientation, and geographic coordinates to create the environment, demonstrating the game's capacity not only to provide the development of abilities, as registered in the experience with the younger students, but also to diagnose the abilities developed along the school years, in the older students' case.

It is important to highlight that to use and seize this teaching methodology it is necessary planning and knowledge of its uses' possibilities, hence the importance of disseminating the results of the research. Among them, the interactivity and immersion features of the projects presented by the students motivate and help them to acquire knowledge, and also stimulate reading and analysis skills and peer interaction, developing the interest in themes that can, apparently, be little attractive in other types of media. 